\title{
Intrinsic Tactile Sensing for the Optimization of Force Distribution in a Pipe Crawling Robot
}

\author{
José A. Gálvez, Pablo González de Santos, and Friedrich Pfeiffer, Senior Member, IEEE
}

\begin{abstract}
This paper describes a tactile sensing system based on a force/torque sensor for the feet of a pipe crawling robot. Such a sensing system is needed for better optimization of force and joint load distribution and a safer avoidance of the risk of foot slippage. While conventional tactile sensing devices typically provide information concerning the spatial distribution of normal pressures, the intrinsic contact sensing system presented in this text only measures the three components of the contact force and two components of the resultant torque. These five parameters are shown to be sufficient to estimate the location of the contact point and hence the orientation of the local contact surface. Such information can then be used by the crawler's control system for the real-time computation of an optimized foot force distribution. The intrinsic tactile sensing method has been experimentally tested on a single leg test setup, while the optimization of force distribution is already functioning in the TUM Pipe Crawling Robot (only with a different, more unripe, sensing system for the contact orientations).
\end{abstract}

Index Terms-Force control, legged locomotion, robot sensing systems, service robots, tactile sensors.

\section{INTRODUCTION}

F ORCE CONTROL is essential for a correct performance of the eight-legged TUM Pipe Crawling Robot, shown in Fig. 1. Tasks of force control in legged robots are the avoidance of the risk of foot slippage, the optimization of the joint load distribution, and, in general, the attainment of the robot's stability, traction, and adaptability. The force control of a walking or crawling machine involves the computation of an optimal force distribution at its footholds. The TUM Pipe Crawling Robot supports itself with at least four legs contacting the tube walls. Each leg has two actuated joints; hence the robot is controlled using at least eight actuators. The force distribution problem is therefore statically indeterminate: for any kinematic stance of the robot, there exists an infinite number of foot force distributions (or, correspondingly, actuator torque combinations) that satisfy the equations of equilibrium. Among them, the crawler's control system may choose an appropriate one according to an on-line optimization scheme.

Manuscript received August 15, 1999; revised June 5, 2000. Recommended by Technical Editor T. Fukuda. The work of J. A. Gálvez was supported in part by the Deutsche Forschungsgemeinschaft (DFG), in part by the Deutscher Akademischer Austauschdienst (DAAD), and in part by the Lehrstuhl B für Mechanik.

J. A. Gálvez was with the Lehrstuhl B für Mechanik, Technische Universität München, Garching, Germany. He is currently with the Instituto de Automática Industrial, CSIC, Madrid, Spain (e-mail: jagalvez@iai.csic.es).

P. González de Santos is with the Instituto de Automática Industrial, CSIC, Madrid, Spain.

F. Pfeiffer is with the Lehrstuhl B für Mechanik, Technische Universität München, 85147 Garching, Germany.

Publisher Item Identifier S 1083-4435(01)02718-1.

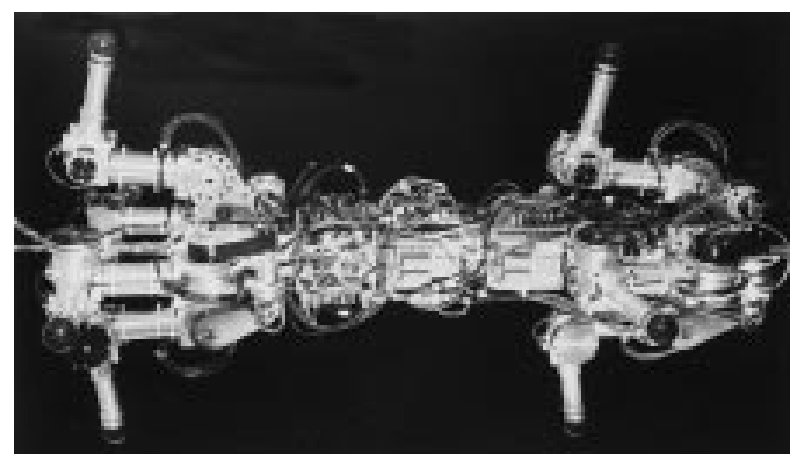

Fig. 1. The TUM pipe crawling robot.

This optimization strategy implements the friction cone inequality constraints that are characteristic of the force distribution problem [5], [15], [18], [21]. The friction cone constraints (actually linearized into friction pyramids) ensure that the adherence conditions are met. The exact placement of the friction cones requires that the normal vectors at the local contact surfaces are known for every foothold. Fig. 2 visualizes the fact that the crawler's feet contact the tube walls at arbitrary orientations. A sensory system is therefore desirable to detect the normal vectors at the robot footholds.

Most of the work dealing with force distribution in legged robots [14]-[16], [18], [19] does not descend to sense and analyze the actual contact under each foot. In other words, though they may formulate the force equilibrium equations together with the friction cone constraints, they do not actually do it in the local support coordinate systems oriented according to the actual surface normals at the points of contact between each foot and the ground. That is the case of walking machines like, e.g., the well-known adaptive suspension vehicle [31] or the MECANT [19]. These machines walk on rough terrain, but their control system implicitly assumes that all local surfaces under each foot are horizontal or parallel to a global support plane. It can be said that they pursue a global approximate minimization of the risk of slippage. While that may be sufficient for a walking machine, it is certainly not for a pipe crawling robot that must support itself in vertical as well as horizontal pipe segments and crawl through curves. In [11], Gardner presented a general formulation of the force distribution problem in walking machines in which the feet contact the ground at arbitrary inclinations. His approach was merely theoretical, though: he outlined a computationally efficient technique to approximate the optimal solution and simulated it. In order to implement a general formulation of the force distribution problem in a real machine, a sensory system is required that, in addition to providing force feedback, also enables the feet to sense contact orientation. In [10], 


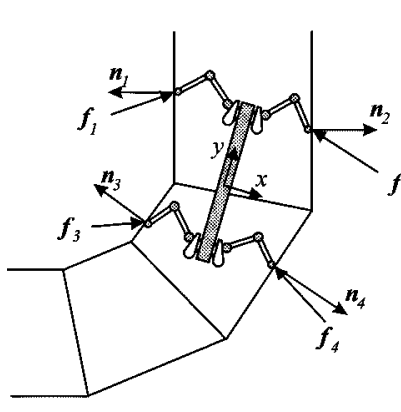

(a)

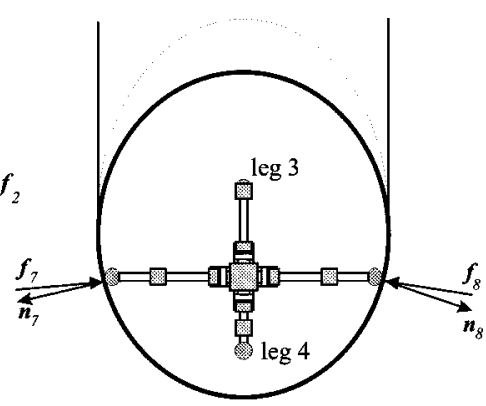

(b)
Fig. 2. Lateral and top drawings of a pipe crawling robot getting into a curve.

the authors report the design of a quadruped walking robot with passive articulated feet that adapt to the ground, thus being able to detect the orientation of the local ground surface by means of two angle sensors integrated at each robot's ankle.

Most tactile sensors, whether based on conductive silicone rubber, pressure-sensitive semiconductors, or piezoelectric elements, detect contact position using surface-mounted arrays of force-sensitive elements [24]. In this paper, a simpler system for the estimation of the normal vectors using a five-axis force/torque sensor is presented. This use of force sensors was first pointed out by Salisbury [30] in the context of manipulation systems. It is usually called intrinsic contact sensing for the use of internal force and torque measurements [1], [2], [6]. Force-based contact sensors have been actually implemented in robotic hands [4], [21], [35] and object shape detection systems [33]. To the authors' best knowledge, no existing legged robots implement this technique.

\section{INTRINSIC TACTILE SENSING Method}

For the purpose of formulating the problem, a six-axis force/torque sensor will be assumed. Later, the limitation that the sensor developed for the TUM Pipe Crawler is in fact a five-axis force/torque sensor will be introduced. It can be accepted that the force originated through the contact between the crawler's foot and the tube wall is a pure force, i.e., no appreciable torques may be exerted. Let $O_{x y z}$ be the reference frame attached to the sensor and $\mathbf{F}$ and $\mathbf{M}$ the measured force and moment in that frame, respectively. The sensor outputs $\mathbf{F}$ and $\mathbf{M}$ are related to the actual contact force $\mathbf{f}$ and the position vector $\mathbf{r}_{c}$ of the contact point by the force and balance equations

$$
\begin{aligned}
\mathbf{F} & =\mathbf{f} \\
\mathbf{M} & =\mathbf{r}_{c} \times \mathbf{f} .
\end{aligned}
$$

The intrinsic contact sensing problem is depicted in Fig. 3. By eliminating $\mathrm{f}$ from (1) and (2), the following expression is obtained:

$$
\mathbf{M}=\mathbf{r}_{c} \times \mathbf{F}
$$

By solving for $\mathbf{r}_{c}$, the line of action of the contact force is given as follows:

$$
\mathbf{r}_{c}=\mathbf{r}_{0}+\lambda \mathbf{F}
$$

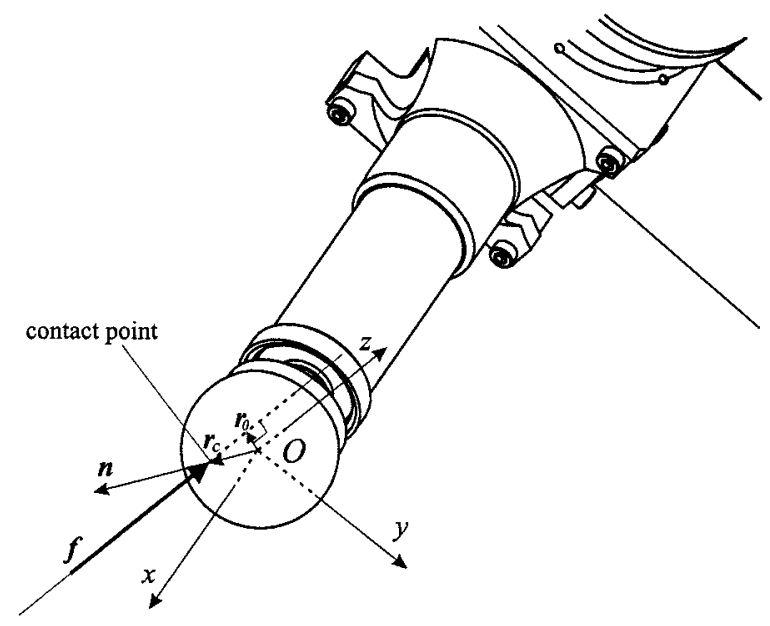

Fig. 3. Problem statement and reference system.

where

$$
\mathbf{r}_{0}=\frac{\mathbf{F} \times \mathbf{M}}{\|\mathbf{F}\|^{2}}
$$

The line of action of the force or wrench axis is a line through $\mathbf{r}_{0}$ and parallel to $\mathbf{F}$ parameterized by $\lambda$. This line intersects the foot surface in two locations: one corresponding to a force pulling out of the surface and one corresponding to a force pushing into the surface. Because adhesive forces are not allowed, the contact point is determined as the intersection point for which the contact force points inwardly at the foot surface, that is

$$
\mathbf{F}^{T} \mathbf{n}<0
$$

where $\mathbf{n}$ is the normal vector at the contact surface (see Fig. 3). If hard contact is assumed, the foot surface geometry can be approximated by a sphere, that is

$$
\left\|\mathbf{r}_{c}\right\|^{2}=R^{2}
$$

where $R$ is the radius of the foot sphere and the origin of the reference system has been placed at the center of the sphere. The normal vector is then related to $\mathbf{r}_{c}$ by

$$
\mathbf{n}=\mathbf{r}_{c} / R
$$

As was mentioned above, only two torque components are measured by the crawler's force sensor. The $x$-component of the torque (i.e., along the sensor's longitudinal axis; see Figs. 3 and 4 ) is not supplied. Therefore, (3) provides a set of only two equations, which together with the equation of the foot surface (7) constitute a system of three equations for the three unknowns $\mathbf{r}_{c}=\left(x_{c}, y_{c}, z_{c}\right)$. This system of equations is determinate as long as the force possesses a nonzero $x$-component. That is no real limitation since the force will rarely have zero $F_{x}$-values and the control system can always set high $F_{x}$-values for the task of the contact orientation measurement. The higher the values of $F_{x}$, the less ill conditioned the system of equations. 


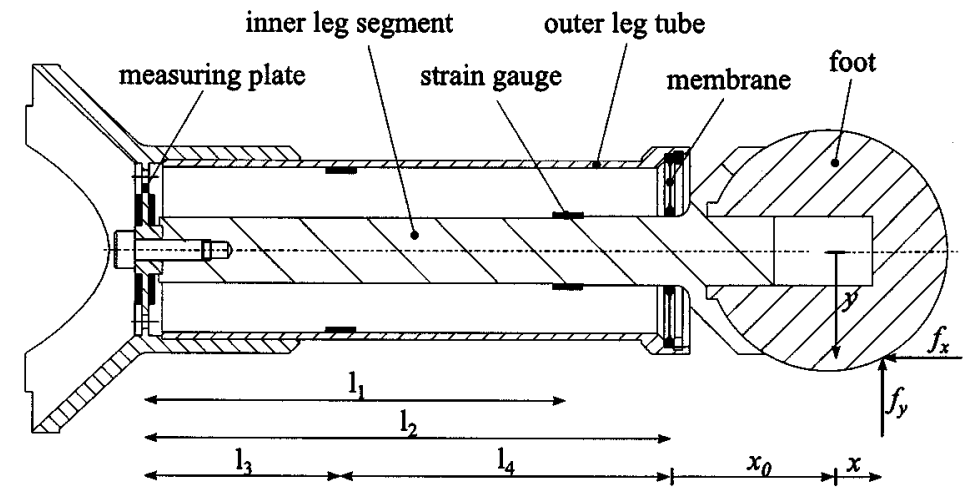

Fig. 4. The 0.15 -m-long force/torque sensor showing eight of the 12 strain gauges; the other four would be seen at the same place of the inner rod and outer tube after a rotation of $90^{\circ}[29]$.

Equations (9)-(14) give the explicit solution of the intrinsic contact sensing problem

$$
x_{c}=\frac{-b \pm \sqrt{b^{2}-4 a c}}{2 a}
$$

where the plus sign is taken when $F_{x}$ is negative and the minus sign otherwise (in fulfilment of condition (6)); and $a, b$, and $c$ are given as follows:

$$
\begin{aligned}
a & =\frac{F_{x}^{2}+F_{y}^{2}+F_{z}^{2}}{F_{x}^{2}} \\
b & =2 \cdot \frac{F_{y} M_{z}-F_{z} M_{y}}{F_{x}^{2}} \\
c & =\frac{M_{y}^{2}+M_{z}^{2}}{F_{x}^{2}}-R^{2} .
\end{aligned}
$$

For the computation of $y_{c}$ and $z_{c}$, the already available value of $x_{c}$ can be used

$$
\begin{aligned}
& y_{c}=\frac{F_{y} x_{c}-M_{z}}{F_{x}} \\
& z_{c}=\frac{F_{z} x_{c}+M_{y}}{F_{x}} .
\end{aligned}
$$

The normal vector at the contact surface can now be found by substituting into (8).

\section{OPTIMIZATION OF FORCE DistRIBUTION}

\section{A. Formulation}

The force distribution problem is the inverse dynamics problem for actively coordinated mechanisms involving multiple frictional contacts with the environment: an appropriate set of joint torques must be determined in order to accomplish a given task or achieve a specified motion in a stable manner (e.g., without slipping). Fig. 2 can be seen as a depiction of the walking and crawling problem for $n$-legs in three-dimensional space. For statically stable systems, the force distribution problem is typically considered separately from the dynamic problem [18]. If the foot contact forces are expressed by means of the components parallel to the body-fixed axes, the equations of force and torque equilibrium can be written in the body-fixed reference frame as follows:

$$
\mathbf{W}=\left[\begin{array}{c}
F_{x}^{\text {ref }} \\
F_{y}^{\text {ref }} \\
F_{z}^{\text {ref }} \\
M_{x}^{\text {ref }} \\
M_{y}^{\text {ref }} \\
M_{z}^{\text {ref }}
\end{array}\right]=\left[\begin{array}{cccc}
\mathbf{I}_{3} & \mathbf{I}_{3} & \cdots & \mathbf{I}_{3} \\
\mathbf{R}_{1} & \mathbf{R}_{2} & \cdots & \mathbf{R}_{m}
\end{array}\right]\left[\begin{array}{c}
{ }^{b} \mathbf{f}_{1} \\
{ }^{b} \mathbf{f}_{2} \\
\vdots \\
{ }^{b} \mathbf{f}_{m}
\end{array}\right]
$$

where

$$
\mathbf{I}_{3}=\left[\begin{array}{lll}
1 & 0 & 0 \\
0 & 1 & 0 \\
0 & 0 & 1
\end{array}\right] \quad \mathbf{R}_{i}=\left[\begin{array}{ccc}
0 & -z_{i} & y_{i} \\
z_{i} & 0 & -x_{i} \\
-y_{i} & x_{i} & 0
\end{array}\right]
$$

For a pipe crawling robot, (15) is read as follows: to support and drive the machine as prespecified, a certain global force and moment must be achieved by the set of forces that the tube walls exert on the feet. $F_{x}^{\text {ref }}, F_{y}^{\text {ref }}$ and $F_{z}^{\text {ref }}$ are the components of the global force reference, i.e., weight and inertial forces (and possibly other external forces); $M_{x}^{\text {ref }}, M_{y}^{\text {ref }}$ and $M_{z}^{\text {ref }}$ are the components of the global torque reference; ${ }^{b} \mathbf{f}_{i}$ are the $3 \times 1$ vectors of foot contact forces (the unknowns of the force distribution problem); $x_{i}, y_{i}$ and $z_{i}$ are the coordinates of the $i$ th foot contact, and $m$ is the number of feet supporting the machine (e.g., four for the particular gait used by the TUM Pipe Crawler).

Equation (15) can be transformed in a way that each foot contact force is expressed in a local coordinate frame with the $x$-axis along the normal to the contact surface. For this purpose, the normals ${ }^{b} \mathbf{n}_{i}$, which are known from Section II, must be used, assuming for brevity that they were already transformed to be expressed in components parallel to the body-fixed axes [left superscript $b$, which will be omitted for simplicity in (18)]

$$
{ }^{b} \mathbf{f}_{i}=\mathbf{C}_{i}{ }^{f} \mathbf{f}_{i}
$$

where

$$
\mathbf{C}_{i}=\left[\begin{array}{ccc}
n_{x i} & \frac{-n_{y i}}{s} & \frac{-n_{x i} n_{z i}}{s} \\
n_{y i} & \frac{n_{x i}}{s} & \frac{-n_{y i} n_{z i}}{s} \\
n_{z i} & 0 & s
\end{array}\right]
$$


with

$$
s=\sqrt{n_{x i}^{2}+n_{y i}^{2}}
$$

In this manner, (15) may be rewritten with the foot contact forces expressed in the surface-normal frames (left supercript $f$ of ${ }^{f} \mathbf{f}_{i}$ )

$$
\mathbf{W}=\left[\begin{array}{cccc}
\mathbf{C}_{1} & \mathbf{C}_{2} & \cdots & \mathbf{C}_{m} \\
\mathbf{R}_{1} \mathbf{C}_{1} & \mathbf{R}_{2} \mathbf{C}_{2} & \cdots & \mathbf{R}_{m} \mathbf{C}_{m}
\end{array}\right]\left[\begin{array}{c}
{ }^{f} \mathbf{f}_{1} \\
{ }_{\mathbf{f}_{2}} \\
\vdots \\
{ }_{f_{\mathbf{f}_{m}}}
\end{array}\right]
$$

Whenever the number of feet in contact $m$ is three or higher, the problem of computing the contact forces that satisfy the equilibrium equations [(15) or (19)] is underspecified. Typical walking robots have three actuated joints per leg, which are thus capable of exerting forces in all three spatial directions. Hence (19) can be used as the equality constraints of an optimization problem with $3 m$ variables, ${ }^{f} f_{x 1},{ }^{f} f_{y 1},{ }^{f} f_{z 1}, \ldots,{ }^{f} f_{x m},{ }^{f} f_{y m}$, and ${ }^{f} f_{z m}$. It was advantageous to transform the equality constraints or equilibrium equations in this way because the inequality cone constraints that prevent slippage are more simply written as functions of the same $3 m$ variables

$$
\frac{\sqrt{{ }^{f} f_{y i}^{2}+{ }^{f} f_{z i}^{2}}}{{ }^{f} f_{x i}} \leq \mu, \quad i=1, \ldots, m
$$

or linearized into friction pyramids that lie entirely within the friction cones (conservative estimate), i.e., defining an effective coefficient of friction $\mu_{\mathrm{eff}}=\mu / \sqrt{2}$

$$
\begin{aligned}
& -\mu_{\mathrm{eff}}^{f} f_{x i}-{ }^{f} f_{y i} \geq 0 \\
& -\mu_{\mathrm{eff}}{ }^{f} f_{x i}+{ }^{f} f_{y i} \geq 0 \\
& -\mu_{\mathrm{eff}}{ }^{f} f_{x i}-{ }^{f} f_{z i} \geq 0 \\
& -\mu_{\mathrm{eff}}{ }^{f} f_{x i}+{ }^{f} f_{z i} \geq 0
\end{aligned}
$$

with $i=1, \ldots, m$. Note that ${ }^{f} f_{x i}$ is always negative when there is contact and that the nonpositivity constraints are already included in (21). The cones could be more accurately represented, at greater computational cost, by pyramids with more than four sides.

It remains to define an objective function, i.e., a function to be minimized or maximized while respecting the equality and inequality constraints. Several methods have been proposed and studied to optimize the force distribution problem [11], [15], [18], [19], though none has ever been implemented in a robot dealing with arbitrarily oriented friction constraints, as is compulsory for our robot. Besides, most of the referred methods appraise too highly the minimization of interaction forces, which can obviously not be the case for a pipe crawling robot that relies on interaction forces to sustain itself within a tube.

Still, our robot has not three actuated joints per leg but two, so that not all three components of the contact forces can be controlled, only two. In the general case, the axes of these two components do not exactly match with those of normal and frictional contact forces. Therefore, the formulation of the optimization problem is not so "clean" as shown above for our robot, because the variables of the optimization problem cannot be the contact forces resolved into the contact surface coordinate system. Anyhow, it must be noted that in practice, the contact normals rarely (or never) diverge more than $30^{\circ}$ from the planes of the actuated forces (the leg planes), as can be viewed in Fig. 2(b). This is important, because high values of the uncontrollable component of the contact force can only be counteracted by setting high values for the normal contact force (or, strictly, for the projection in the leg plane of the normal contact force), so that the contact force vector lies within the allowed pyramidal region.

\section{B. Implementation [27]}

Various papers in the 1980s and early 1990s dealt with computational efficiency of algorithms for force distribution [5], [11], [17], sometimes forsaking exact and/or general optimality in favor of runtime-saving approximations to the optimal solutions. Some years have passed, computers have experienced enormous progress, and the truth is that no great problems were encountered for implementing optimization routines capable of real-time operation in the TUM Pipe Crawler [27] (an approximation is indeed used: friction pyramids instead of cones for the inequality constraints). A dual Pentium II PC running a Linux operating system whose kernel was modified for real-time functionality was employed for the task. The optimization routines (see below) were either in-house programmed or slightly modified from the Numerical Recipes Software [26]. The relevant information about computing times is that they were always less than $40 \mathrm{~ms}$, which is sufficient for the refreshing of force setpoints with real-time operability for a machine that changes its kinematic stance with a maximum speed of $0.1 \mathrm{~m} / \mathrm{s}$. The cycle time for the force feedback control loop is $2 \mathrm{~ms}$. It can be said that real-time efficiency is, with present computers, no longer a great concern for the optimization of force distribution with linearized constraints by problems with the complexity of the one described here.

For describing the force distribution problem of our particular robot, it is convenient to come back to (15), in which the foot forces were expressed in the body-fixed coordinate system. In this reference system, the controllable foot contact forces $\left({ }^{b} f_{x i}\right.$ and $\left.{ }^{b} f_{y i}\right)$ and the uncontrollable foot contact forces $\left({ }^{b} f_{z i}\right)$ are directly given, because the robot's gait consists of alternating the two leg planes (of four legs each) and the body's $z$-axis can be alternately changed to be perpendicular to the supporting legs' plane. Let us define a vector $\mathbf{f}_{c}$ containing all $2 m$ controllable forces $\left({ }^{b} f_{x 1},{ }^{b} f_{y 1}, \ldots,{ }^{b} f_{x m},{ }^{b} f_{y m}\right)$ and a vector $\mathbf{f}_{u}$ containing the $m$ uncontrollable forces $\left({ }^{b} f_{z 1}, \ldots,{ }^{b} f_{z m}\right)$. The $4 m$ inequality constraints given by (21) are easily transformed to obtain, finally, the linear inequality constraints that ensure that force vectors lie within the allowed pyramidal regions

$$
\mathbf{G}_{c} \mathbf{f}_{c}+\mathbf{G}_{u} \mathbf{f}_{u} \geq \mathbf{0}
$$

where the $4 m \times 2 m$ matrix $\mathbf{G}_{c}$ and the the $4 m \times m$ matrix $\mathbf{G}_{u}$ are obtained from the system of (21) after a linear transformation into the body-fixed reference system. The value of $m$ is four for the gaits used by the TUM Pipe Crawling Robot. 
For the same reason, only rows 1, 2, and 6 from (15) have to be considered by our optimization problem. Therefore, the equality constraints are as follows:

$$
\mathbf{W}_{c}=\left[\begin{array}{c}
F_{x}^{\mathrm{ref}} \\
F_{y}^{\mathrm{ref}} \\
M_{z}^{\mathrm{ref}}
\end{array}\right]=\mathbf{A f}_{c}
$$

with

$$
\mathbf{A}=\left[\begin{array}{ccccc}
1 & 0 & \cdots & 1 & 0 \\
0 & 1 & \cdots & 0 & 1 \\
-y_{1} & x_{1} & \cdots & -y_{m} & x_{m}
\end{array}\right]
$$

Another source of inequality constraints is the limits on the torques that can be exerted by the actuators

$$
-T_{j, \max } \leq T_{j} \leq T_{j, \max }, \quad j=1, \ldots, 2 m
$$

Since the effect of the legs' masses can be neglected, the torques are directly related to the contact force components within the leg plane by the $2 \times 2$ Jacobians of the $m$ subchains (i.e., the $m$ legs), $\mathbf{J}_{1}, \ldots, \mathbf{J}_{m}$, which for simplicity will be collected in matrix $\mathbf{J}$

$$
\mathbf{T}=\left[\begin{array}{c}
T_{1} \\
\vdots \\
T_{2 m}
\end{array}\right]=\left[\begin{array}{ccc}
\mathbf{J}_{1}^{T} & \cdots & \mathbf{0} \\
& \ddots & \\
\mathbf{0} & \cdots & \mathbf{J}_{m}^{T}
\end{array}\right] \mathbf{f}_{c}=\mathbf{J}^{T} \mathbf{f}_{c} .
$$

Given that adherence conditions need only be safely fulfilled (there is not a better performance if a certain related index is minimized) and a faultless performance is anyhow the most determinant question for this application (more than, e.g., energy consumption), the following min-max approach was chosen for the objective function:

$$
\max \left\{\frac{\left|T_{1}\right|}{T_{1, \text { lim }}}, \frac{\left|T_{2}\right|}{T_{2, \text { lim }}}, \ldots, \frac{\left|T_{2 m}\right|}{T_{2 m, \text { lim }}}\right\} \rightarrow \min !
$$

where $T_{j, \mathrm{lim}}$ is the nominal maximum torque for the $j$ th joint (a positive number).This criterion is most of all justified in consideration of the thermic properties of electrical motors: within the overload region, motors can work for a reasonable amount of time as long as the overload is "reasonable" too. The constraints have to be stated in function of the torques by substituting (26) into (22) and (23).

Min-max problems with linear constraints can be transformed into linear programming problems [12]. For the min-max problem defined by (22), (23), (26), and (27), the equivalent linear problem is as follows, with $k$ and the $2 m \times 1$ vector of scaled joint torques $\mathbf{T}^{*}$ being the optimization variables:

$$
\begin{aligned}
k & \longrightarrow \max ! \\
\mathbf{A J T}^{*}-k \mathbf{W}_{c} & =\mathbf{0} \\
\mathbf{G}_{c} \mathbf{J T}^{*}+k \mathbf{G}_{u} \mathbf{f}_{u} & \geq \mathbf{0} \\
\mathbf{T}^{*} & \leq \mathbf{T}_{\lim } \\
\mathbf{T}^{*} & \geq-\mathbf{T}_{\lim }
\end{aligned}
$$

where $\mathbf{T}_{\lim }$ is the vector containing all $T_{j, \mathrm{lim}}$ (positive numbers).
The setpoints for the torques stem from the optimal $k=k_{\mathrm{opt}}$ obtained by the optimization

$$
\mathbf{T}=\frac{1}{k_{\mathrm{opt}}} \mathbf{T}^{*}
$$

Optimal contact forces can be obtained from the optimal contact torques by substituting in (26).

A good quality of this approach is that (25) does not apply as an inequality constraint, because in fact the torques are the variables being minimized, so that an overload of actuators is allowed if strictly necessary $\left(k_{\mathrm{opt}}<1\right)$.

The simplex algorithm-specifically, the routines from [26] — was used to solve this linear programming problem. As already shown in [15] within the same context (optimization of force distribution) and verified by simulations and experiments within the TUM Pipe Crawler's project [27], [29], linear programming solutions experience a slight nonsmooth behavior over time, even if the constraints vary smoothly and slowly. This is the well-known phenomenon of "alternate optima" in linear programming: the simplex algorithm returns a vertex of a polyhedron as its answer and, as constraints change over time (even if they change smoothly), discontinuities occur when answers jump from one vertex to another. These discontinuities are not critical in other fields, but have undesirable effects in the stability of feedback controllers if they have to be fed with such a nonsmooth sequence of setpoints.

The problem is completely eliminated by attaching an additional quadratic term to the objective function that penalizes variations of the joint torques between two successive instants in time

$$
-k+\left(\Delta \mathbf{T}^{* T} \mathbf{C} \boldsymbol{\Delta} \mathbf{T}^{*}\right) \rightarrow \min !
$$

with

$$
\Delta \mathbf{T}^{*}=\mathbf{T}^{*}-\mathbf{T}_{\text {old }}^{*}
$$

and with the same constraints (29)-(32) as for the linear programming problem. $\mathbf{T}_{\text {old }}^{*}$ is the result of the previous optimization and $\mathbf{C}$ is a $2 m \times 2 m$ positive-definite weighting matrix.

This quadratic optimization problem is transformed into a linear complementarity problem and then solved with Lemke's algorithm [20] (see, e.g., [7] if preferred). The purpose is to obtain the same results as with linear programming, but without the abrupt slight changes over time. This is achieved by choosing values as small as possible for the elements of $\mathbf{C}$, but not so small as to not repress potential changes in the calculated torques caused by noise in the sensors' signals. The constraints of the optimization problem vary with changes in the signals supplied by the force sensors (specifically with $\mathrm{f}_{u}$, which is an input to the optimization routine) and the joint angle sensors; hence the calculated optimal torques are affected by noise. For further details and experimental results on the optimization of force distribution in the TUM Pipe Crawling Robot, see [27] and [29]. 


\section{TUM PIPE CRAWLING ROBOT IN BRIEF}

Different robots have been developed worldwide for inspecting or repairing tubes from inside [8], [9], [22], [23], [32]. The TUM Pipe Crawling Robot (see Fig. 1) has eight legs driven by two dc motors each. It weighs about $20 \mathrm{~kg}$ and is able to walk and crawl within pipes with diameters of about 60-70 $\mathrm{cm}$. The leg design [27] is based on that of the hexapod TUM Walking Machine [25].

The robot is controlled by five on-board 16-bit microcontrollers that communicate over a Controller Area Network (CAN) - an ISO standard for real-time serial data communication. Gravity, velocity, and gear friction observers, gait pattern, task distribution, and, in general, the realization of the central and decentralized control levels (each one of them simultaneously concerned with coordination and operating levels) are described in [27]-[29]. In the decentralized operating level, the actual force feedback control is realized, namely, based on the nonlinear control method of feedback linearization [27].

The computation of the force setpoints takes place in the central operating level. The forces are distributed among the legs under consideration of the friction limits and minimizing the maximal joint torque $T_{j}$ referred to the maximal possible torque $T_{j, \max }$ of the respective joint, as described in Section III-B. Since the 16-bit microcontrollers have no good floating-point performance, this is the only task that must be done by another type of microprocessor. At present, this is done by an external PC that is also connected to the five on-board microcontrollers over a CAN interface. In the future, this should be done using an on-board digital signal processor.

To date, the contact normals are estimated by touching the surroundings of the contact point at different positions and then calculating the least squares approximation for the contact plane [27]. This not only is a slow sensing system but also is inaccurate. Note that not just the joint angle sensors of the touching leg are involved in this measuring process, but also those of the four legs supporting the robot. It is apparent that a better sensing system is needed for this task, and this was in fact the motivation for the work presented in this paper.

\section{FORCE/TORQUE SENSOR}

For the range of $\pm 300 \mathrm{~N}$, commercial force sensors were either too expensive or too unwieldy or unsuitable for static measurements due to high drift. Therefore, a special strain-gaugebased force sensor was developed for the TUM Pipe Crawling Robot [29]. Strain gauge transducers are the most widely used sensors for force measurement. They have high sensitivity and measurement accuracy and require relatively simple amplifiers. Their main drawback is sensitivity to temperature, which can be counteracted by the appropriate use of full Wheatstone bridges, as is done by our force sensor. Good books for understanding the different aspects to consider in the design of this type of sensors are, e.g., [3] and [13].

The sensor is integrated in the leg's outer segment, as shown in Fig. 4, which is a longitudinal section of the three-dimensional Fig. 3. The real difficulty by the design of the force sensor is the measurement of longitudinal forces, i.e., $f_{x}$. Transverse forces $f_{y}$ and $f_{z}$ are more easily measured because they are di- rectly reflected in the bending of the inner rod and outer tube. Since elastic deformations caused by transverse forces are much higher than the ones caused by longitudinal ones, much care was taken at the design stage to uncouple the measurement of transverse and longitudinal forces, i.e., to limit the negative effect of crosstalks. That was achieved through the mechanical design explained in the next paragraph.

Within the small range of deformations occurring in the structure, the $0.1-\mathrm{mm}$-thick membrane acts as an spherical and prismatic passive joint: the inner rod can move axially and rotate with respect to the outer tube, but cannot shift along radial directions. Longitudinal forces are thus straight directed into the measuring plate (see Fig. 4). By using a full bridge of four strain gauges appropriately arranged at the measuring plate (again, see Fig. 4) and laboriously tuning the individual sensitivities of each strain gauge with the help of additional resistances connected in series and in parallel to each strain gauge [27] (see also [3] for the theory of Wheatstone bridges), we achieve the result that possible torques and transverse forces introduced in the measuring plate have a negligible effect on the measurement of longitudinal forces. In this manner, the effect of crosstalks on the measurement of forces could be limited to less than $0.5 \%$ in all cases.

The designer also took much care that the mechanical construction was not a source of hysteresis in the sensor. For this reason, axial bearings and bushings were rejected in favor of the membrane. Axial bearings and bushings would introduce friction forces in the structure and, therefore, hysteresis. The membrane was dimensioned to absorb radial loads with no trouble, but simultaneously having very high flexibility in axial direction. This permits small axial displacements of the inner rod with respect to the outer tube at the expense of small linear elastic deformations of the membrane, which is the best way to avoid the hysteresis caused by mechanical factors. This way, hysteresis-related errors in force measurements are limited to less than $1 \mathrm{~N}$, i.e., less than $1 \%$ if a range of $100 \mathrm{~N}$ is taken [27], [34].

On the inner rod, four strain gauges are located as two half-bridges, which are able to determine the two components of the bending torque. The outer tube has as well two pairs of strain gauges (see Fig. 4; inclusive explanation in caption). The bending torques $T$ (index ot for outer tube and index ir for inner rod) and the corresponding output of the two half-bridges $U$ in plane $x y$ (paper plane in Fig. 4) are given by the following equations:

$$
\begin{aligned}
& U_{\mathrm{ot}}=k_{\mathrm{ot}} T_{\mathrm{ot}}, \quad \text { with } T_{\mathrm{ot}}=\frac{l_{4}}{l_{2}}\left[f_{x} y-f_{y}\left(x-x_{0}\right)\right]-f_{y} l_{4} \\
& U_{\mathrm{ir}}=k_{\mathrm{ir}} T_{\mathrm{ir}}, \quad \text { with } T_{\mathrm{ir}}=\frac{l_{1}}{l_{2}}\left[f_{x} y-f_{y}\left(x-x_{0}\right)\right] .
\end{aligned}
$$

By building a full bridge with the four strain gauges in plane $x y$ (two on the inner rod and two on the outer tube) and appropriately tuning the amplification factor $k_{\mathrm{ir}}$, the force in $y$-direction can be directly given, independently of the actual contact point on the foot

$$
k_{\mathrm{ir}}=\frac{l_{4}}{l_{1}} k_{\mathrm{ot}} \rightarrow f_{y}=\frac{U_{\mathrm{ir}}-U_{\mathrm{ot}}}{k_{\mathrm{ot}} l_{4}}=k_{y}\left(U_{\mathrm{ir}}-U_{\mathrm{ot}}\right) .
$$

The "tuning" of $k_{\mathrm{ir}}$ is done with the help of additional resistances in series with the inner rod strain gauges, as described in [27]. 
With the same method, the lateral force in $z$-direction is determined (using the strain gauges on the $x z$-plane). In this way, a three-axes force sensor is obtained. Total errors in force measurements are smaller than $2 \%$ in all cases, which is sufficient for the force control of legged robots.

In order to implement the intrinsic tactile sensing method presented in this paper, two additional half-bridges were built with the strain gauges on the inner rod, so that the torques in $y$ and $z$-axes caused by the contact forces can be separately measured. Therefore, the sensor supplies five signals: two given by (37) and (38), two by the equivalent equations for the $x z$-plane, and a fifth signal proportional to the longitudinal force $f_{x}$ measured by means of the four strain gauges on the measuring plate.

Since the mechanical structure is rigid enough and the force/torque sensor is mounted very close to the leg's foot, a quasi-static relation between the force applied and the strain measurements is achieved. The signals given by the force sensor are reset to zero each time the leg is in transfer phase (i.e., the foot on the air), and contact is detected when the measured force is higher than $5 \mathrm{~N}$. Drift-related errors are negligible for the range of time periods between resets. The dynamic response of the sensor can be considered to be instantaneous, and therefore detection of contact can be done flawlessly.

\section{EXPERIMENTAL DESIGN}

For the purpose of testing the feasibility of an implementation of the intrinsic tactile sensing method in the pipe crawling robot, a single-leg test setup was used. A picture of this setup is shown in Fig. 5. The single leg can contact the surface below at different points and hence at different angles within the leg plane. To allow the contact surface to be other than the horizontal (that is, to let the normal vector have a third component orthogonal to the leg plane), an additional montage was built that permits an aluminum plate to be adjusted at a desired angle above the horizontal [see also Fig. 6(b)].

The leg's microcontroller communicates with an external PC through a CAN interface, which allows the user to get informations about the system and give control commands. The experiments were carried out by sending from the PC incremental position setpoints. This way the leg can be led to contact the surface at different positions. Once the contact is established, the intrinsic tactile sensing problem is solved in real time by the microcontroller as described in Section II, employing the force and torque signals given by the sensor. This way, contact orientation is computed on-line, and the required data are simultaneously sent to the PC. During the time when the leg is in contact with the surface, the position setpoints can be slightly changed (still maintaining contact) from the PC in order to make the contact force change its direction and test if the results are thereby affected.

Two angles were employed to assess the accuracy of the sensing system:

1) angle $\gamma$ formed by the leg's outer segment and the vertical [see Fig. 6(a)];

2) angle $\psi$ at which the aluminum plate is adjusted over the horizontal plane [see Fig. 6(b)].

The microcontroller software was programmed to estimate the values of $\gamma$ and $\psi$ and send the estimations to the external

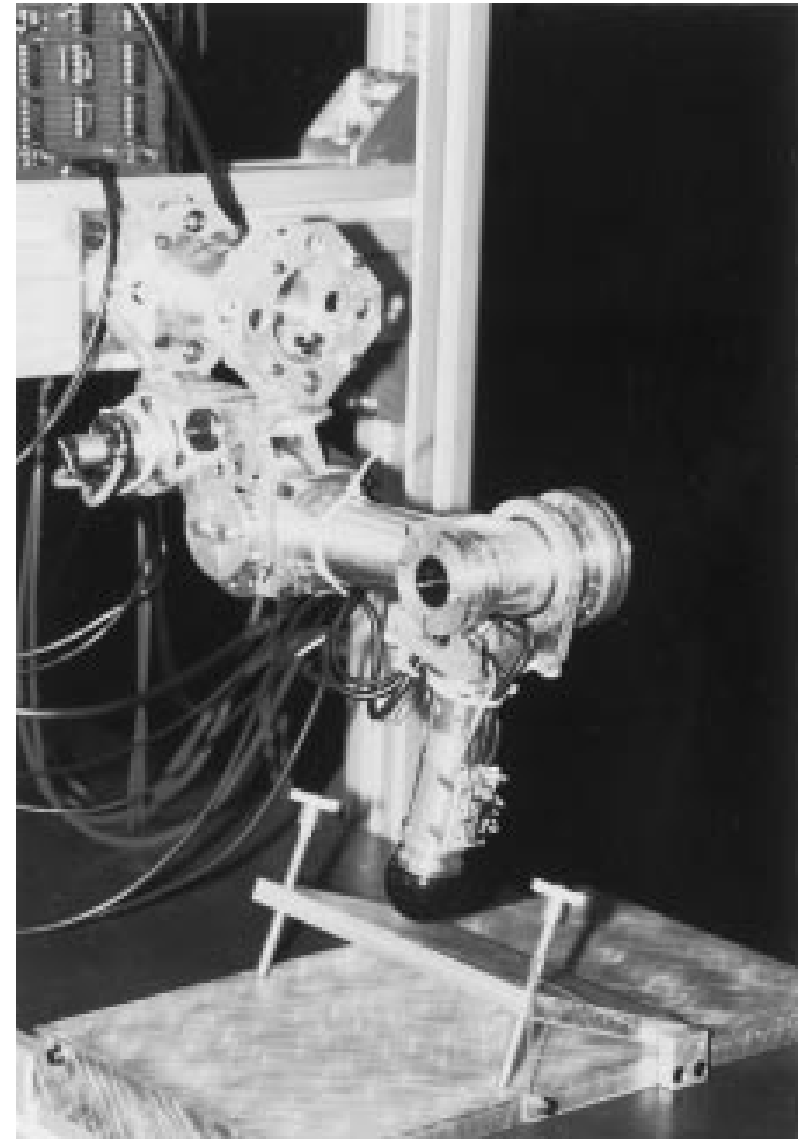

Fig. 5. Single-leg test setup.

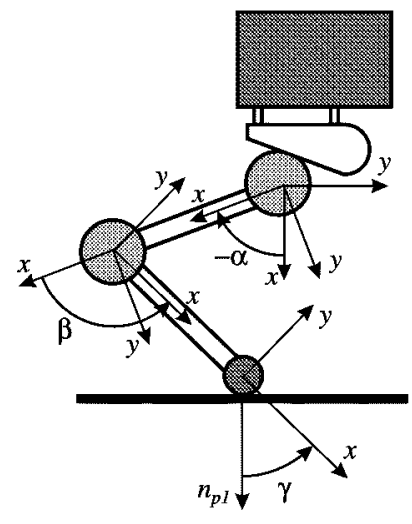

(a)

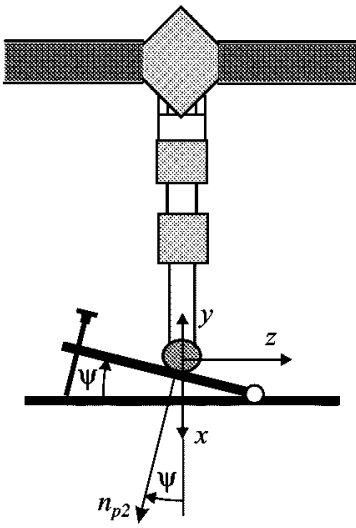

(b)
Fig. 6. Two views of the single-leg test setup showing angles $\gamma$ and $\psi$. The subscripts in $n_{p 1}$ and $n_{p 2}$ indicate that the projections of the normal vector in the paper plane are meant.

PC. These two angles were chosen because they are the two parameters that permit direct comparison of the test results with the external arrangement. The angle $\psi$ of the adjustable aluminum plate was varied from $-30^{\circ}$ to $+30^{\circ}$ in steps of $10^{\circ}$. For each value of $\psi$, the leg was commanded to contact the surface at different angles $\gamma$, which can be measured not only by means of the force/torque sensor but also making use of the leg's joint angle sensors $[\gamma=\alpha+\beta$, as seen in Fig. 6(a)]. The measure- 

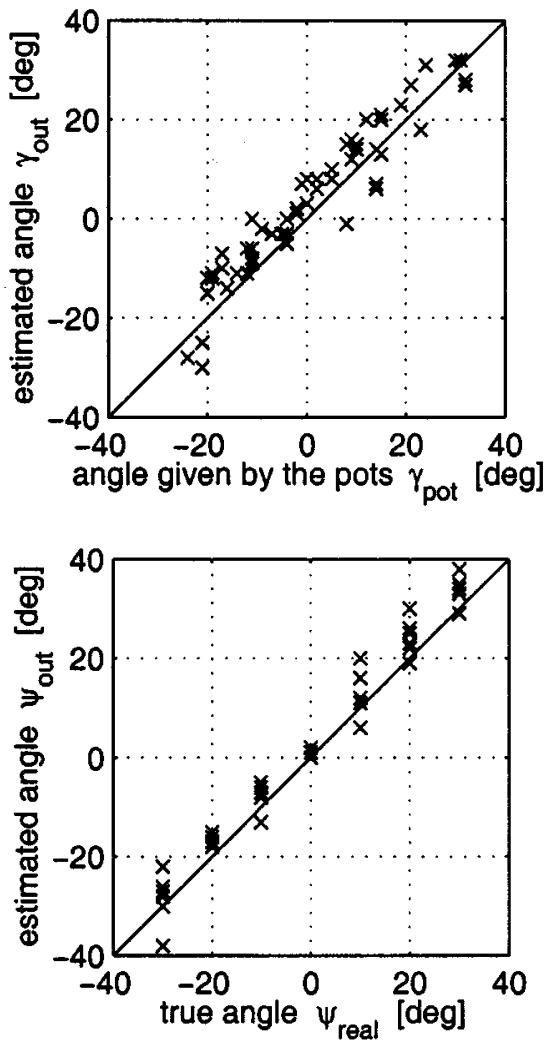

Fig. 7. Experimental results-estimated contact angles using the force-based sensing system.

ment range was $150 \mathrm{~N}$ for the force and $3.5 \mathrm{Nm}$ for the torque. The relation between angles $\gamma$ and $\psi$ and vector quantities $\mathbf{n}$ and $\mathbf{r}_{c}$ (calculated as described in Section II) is given by the following equations:

$$
\begin{aligned}
& \gamma=-\arcsin \left(\frac{n_{y}}{\sqrt{n_{x}^{2}+n_{y}^{2}}}\right)=-\arcsin \left(\frac{y_{c}}{\sqrt{x_{c}^{2}+y_{c}^{2}}}\right) \\
& \psi=-\arcsin \left(n_{z}\right)=-\arcsin \left(\frac{z_{c}}{R}\right) .
\end{aligned}
$$

\section{EXPERIMENTAL RESULTS}

In Fig. 7, the results of the series of experiments described in Section VI are shown, providing an overall impression of the accuracy and feasibility of the sensing system. In the upper graphic, the computed values of $\gamma$ are plotted versus the values of $\gamma$ given by the leg's joint angle sensors, each point of the graphic corresponding indistinctly to values of $\psi$ between -30 and $30^{\circ}$. These values of $\psi$ can be seen in the lower graphic, which shows the estimated values versus the true angles fitted with the adjustable aluminum plate (the points of this graphic correspond likewise to the different values of $\gamma$ that can be seen in the upper one). All values of angles $\gamma$ and $\psi$ were determined by solving the intrinsic tactile sensing problem formulated in Section II, with the contact forces being those resulting from the interaction. These forces have, as described in Section VI, both tangential and normal components, indistinctly, like they have during normal operation of the crawler (not overlooking that there must always be a longitudinal component for our five-axis sensor to be able to unequivocally solve the system of equations, which can be watched over by the control system, as explained in Section II).

During the feasibility tests reported here, the same calibration factor was taken for both negative and positive torques, following the calibration model taken for the forces beforehand. However, it was later noticed that the factors are slightly different for negative and positive torques. Consequently, the accuracy improves if a calibration line is taken for positive torques and another one for negative ones. Thereby, the cloud of points in Fig. 7 would be more equally distributed among both sides of the identity line (our sensor has a noteworthy drawback of the mechatronic kind: it is laborious to calibrate).

The observable maximal errors of $9^{\circ}$ correspond to maximal errors of $2.7 \mathrm{~mm}$ on the foot surface. Frictional and normal forces are calculated using the transpose of matrix $\mathbf{C}_{i}$, given by (18), with maximal errors of $15 \%$. This might seem like really big errors, but it is actually good enough for what this estimate is used for: the formulation of the constraints for the optimization problem described in Section III. To realize this, note that the approximation of formulating friction pyramids instead of friction cones introduces a maximal error of an even greater magnitude: $29 \%$ if the pyramids are four-sided. This approximation is adopted by all previous works dealing with force distribution that formulate friction constraints [5], [15], [18]. The friction factor $\mu$ is also not known with total confidence. With these approximations a conservative estimate is taken, which must also take into account the possible maximal inaccuracies of $15 \%$ in the estimates of frictional and normal forces. In addition to calibration errors, other sources of error are precision errors in the run-time measurements of forces and torques, errors in the geometrical model of the foot surface, and deformations of the foot during contact. Finally, and most significantly, all these errors are magnified by the inherent ill condition of the system of equations that is to be resolved by the force-based tactile sensing algorithm.

\section{CONCLUSION}

In this paper, a method for the estimation of the normal vectors at the footholds of a pipe crawling robot using force/torque information is presented. The work was motivated by the need of a sensory system for the correct statement of the friction inequality constraints that are used for the robot's control. First, the mathematical and mechanical principles of intrinsic tactile sensing are formulated. Next, the force distribution problem is depicted and the implementation of a successful optimization algorithm to solve it is described. On this subject, the friction constraints are stated in the local support coordinate systems oriented according to the surface normals at the robot footholds. Then, the TUM Pipe Crawling Robot and its special force/torque sensor are described. Finally, experiments applying the presented theory of intrinsic tactile sensing on a single-leg test setup are reported. Estimated values of the contact orientation can be obtained with maximal errors of $9^{\circ}$ not depending on either the contact point or the direction 
of the contact force. A natural future extension of this work is the introduction of a model for the elastic deformations of the rubber foot subjected to a certain contact interaction. This model will then be used for the more accurate formulation of the foot surface geometry that is employed in the intrinsic contact sensing algorithm.

\section{ACKNOWLEDGMENT}

The authors wish to thank G. Mayr, who did the electronic work, K. Löffler, who lightened the informatic difficulties, and T. Rossmann, who designed both mechanics and control of the TUM Pipe Crawling Robot.

\section{REFERENCES}

[1] A. Bicchi, "Intrinsic contact sensing for soft fingers," in Proc. IEEE Int. Conf. Robotics and Automation, Cincinatti, OH, 1990, pp. 968-973.

[2] A. Bicchi, K. Salisbury, and D. L. Brock, "Contact sensing from force measurements," Int. J. Robot. Res., vol. 12, no. 3, pp. 249-262, 1993.

[3] G. M. Bray, G. Barbato, and R. Levi, Theory and Practice of Force Measurements. New York: Academic, 1990.

[4] D. L. Brock and S. Chiu, "Environment perceptions of an articulated robot hand using contact sensors," in Proc. ASME Winter Meeting, Miami, FL, 1985, pp. 89-96.

[5] F. T. Cheng and D. E. Orin, "Efficient algorithm for optimal force distribution-The compact-dual LP method," IEEE Trans. Robot. Automat., vol. 6, no. 2, pp. 178-187, 1990.

[6] P. Dario, "Tactile sensing for robots: Present and future," in The Robotics Review 1, O. Khatib, J. J. Craig, and T. Lozano-Pérez, Eds. Cambridge, MA: MIT Press, 1989, pp. 133-146.

[7] R. Fletcher, Practical Methods of Optimization, 2nd ed. New York: Wiley, 1987.

[8] T. Fukuda, K. Kurashige, and F. Arai, "Recent topics on robotic actuation technologies," in Proc. 2nd Int. Conf. Climbing and Walking Robots (CLAWAR 99), Portsmouth, U.K., 1999, pp. 3-15.

[9] T. Fukuda, M. Uemura, and H. Hosokai, "Rubber gas actuator driven by hydrogen storage alloy for in-pipe inspection mobile robot with flexible structure," Proc. IEEE Int. Conf. Robotics and Automation, vol. 3, pp. $1847-1852,1989$.

[10] J. A. Gálvez, P. González de Santos, and M. Armada, "A force controlled robot for agile walking on rough terrain," in Proc. IFAC Int. Workshop Intelligent Components for Vehicles, Sevilla, Spain, 1998, pp. 247-252.

[11] J. F. Gardner, "Efficient computation of force distributions for walking vehicles on rough terrain," Robotica, vol. 10, pp. 427-433, 1992.

[12] P. Gill, W. Murray, and M. Wright, Practical Optimization, London, U.K.: Academic, 1981

[13] D. M. Gorinevsky, A. M. Formalsky, and A. Y. Schneider, Force Control of Robotics Systems. New York: CRC Press, 1997.

[14] D. M. Gorinevsky and A. Y. Schneider, "Force control in locomotion of legged vehicles over rigid and soft surfaces," Int. J. Robot. Res., vol. 9, no. 2, pp. 4-23, 1990.

[15] C. A. Klein and S. Kittivatcharapong, "Optimal force distribution for the legs of a walking machine with friction cone constraints," IEEE Trans. Robot. Automat., vol. 6, pp. 73-85, 1990.

[16] C. A. Klein, K. W. Olson, and D. R. Pugh, "Use of force and attitude sensors for locomotion of a legged vehicle over irregular terrain," Int. J. Robot. Res., vol. 2, no. 2, pp. 3-17, 1983.

[17] V. Kumar and K. J. Waldron, "Suboptimal algorithms for force distribution in multifingered grippers," IEEE Trans. Robot. Automat., vol. 5, pp. 90-99, 1989.

[18] _ _Force distribution in walking vehicles," Trans. ASME J. Mech. Design, vol. 112, pp. 90-99, 1990.

[19] H. Lehtinen, "Force based motion control of a walking machine," Ph.D. dissertation, Tech. Res. Center of Finland, 1994.

[20] C. E. Lemke, "Bimatrix equilibrium points and mathematical programming," Manage. Sci., vol. 11, pp. 681-689, 1965.

[21] M. T. Mason and J. K. Salisbury, Robot Hands and the Mechanics of Manipulation. Cambridge, MA: MIT Press, 1985.

[22] F. Miller, "Kanalarbeiter aus Gummi, Elektronik und Stahl," Der Fraunhofer, pp. 4-8, 1993.
[23] W. Neubauer, "A spider-like robot that climbs vertically in ducts or pipes," in Proc. Int. Conf. Intelligent Robots and Systems, vol. 2, Munich, Germany, 1994, pp. 1178-1185.

[24] H. R. Nicholls and M. H. Lee, "A survey of robot tactile sensing technology," Int. J. Robot. Res., vol. 8, no. 3, pp. 3-30, 1989.

[25] F. Pfeiffer, J. Eltze, and H.-J. Weidemann, "The TUM-walking machine," Intell. Automat. Soft Comput., vol. 1, pp. 307-323, 1995.

[26] H. P. Press, S. A. Teukolsky, W. T. Vetterling, and B. P. Flannery, Numerical Recipes in Fortran: The Art of Scientific Computing, 2nd ed. Cambridge, U.K.: Cambridge Univ. Press, 1992.

[27] T. Roßmann, Eine Laufmaschine für Rohre, ser. 8. Düsseldorf, Germany: VDI-Verlag, 1998

[28] T. Roßmann and F. Pfeiffer, "Control and design of a pipe crawling robot," in Proc. 13th IFAC World Congress Automatic Control, San Francisco, CA, 1996, pp. 465-470.

[29] —-, "Control of a pipe crawling robot," in Proc. Euromech 375, Biology and Technology of Walking, Munich, Germany, 1998, pp. 133-140.

[30] J. K. Salisbury, "Interpretation of contact geometries from force measurements," in Proc. 1st Int. Symp. Robotics Research, Bretton Woods, $\mathrm{NH}, 1983$, pp. 1-6.

[31] S. M. Song and K. J. Waldron, Machines That Walk: The Adaptive Suspension Vehicle. Cambridge, MA: MIT Press, 1989.

[32] K. Suzumori, T. Miyagawa, M. Kimura, and Y. Hasegawa, "Micro inspection robot for 1-in pipes," IEEE/ASME Trans. Mechatron., vol. 4, pp. 286-292, 1999.

[33] T. Tsujimura and T. Yabuta, "Object detection by tactile sensing method employing force/torque information," IEEE Trans. Robot. Automat., vol. 5, pp. 444-450, 1989.

[34] R. Wolf, "Ermittlung des Betriebsparameter eines Rohrkrabblerbeins," Lehrstuhl B für Mechanik, Tech. Univ. München, Munich, Germany, 1996.

[35] X. Zhou, Q. Shi, and Z. Li, "Contact localization using force/torque measurements," in Proc. IEEE Int. Conf. Robotics and Automation, vol. 2, 1996, pp. 1339-1344.

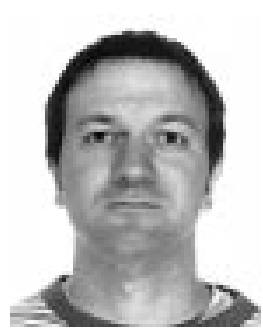

José A. Gálvez studied mechanical engineering at the Universidad de Zaragoza, Zarazoga, Spain, and the Technische Universität Braunschweig, Germany, with an exchange program. He graduated from the Universidad de Zaragoza, Zarazoga, Spain, in 1995.

He worked in the automotive supply industry as a Development Engineer for German multinational Behr in Barcelona and Stuttgart (1995-1996). Since 1996, he has been a Predoctoral Research Assistant at the Instituto de Automática Industrial of the Consejo Superior de Investigaciones Científicas (Spanish Council for Scientific Research, CSIC), Madrid, Spain. . There, he has been engaged in the development of the SILO4 walking robot, a replica of which he assisted in taking its first steps at the Laboratoire de Vision et Robotique of Bourges, France (2000). In 1998-1999, he was on a 13-month research stay at the Lehrstuhl B für Mechanik of the Technische Universität München, Germany.

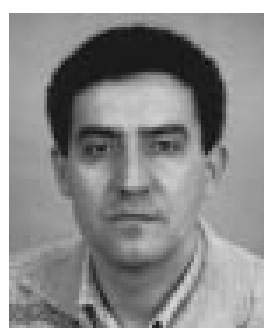

Pablo González de Santos received the Ph.D. degree from the University of Valladolid, Spain, in 1986.

$\mathrm{He}$ is a Research Scientist with the Spanish Council for Scientific Research (CSIC). Since 1981, he has been actively actively in the design and development of industrial robots and in special robotic systems. His work during last ten years has been focused on walking machines. He worked on the AMBLER project as a Visiting Scientist at the Robotics Institute of Carnegie Mellon University, Pittsburgh, PA. Since then, he has been leading the development of several walking robots, such as the RIMHO robot designed for intervention in hazardous environments, the ROWER walking machine developed for welding tasks in ship erection processes, and the SILO4 walking robot intended for educational and basic research purposes. He has also participated in the development of other legged robots such as the REST climbing robot and the TRACMINER. He is now working on applying walking machines to the field of humanitarian demining. 


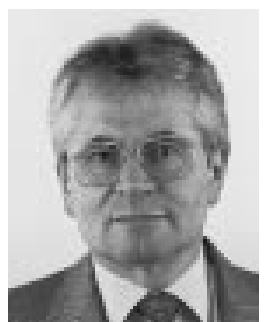

Friedrich Pfeiffer (SM'91) received the Dipl.-Ing. degree in mechanical engineering and the Dr.-Ing. degree in aerodynamics from the Technical University of Darmstadt, Darmstadt, Germany, in 1961 and 1965 , respectively.

He was a Research Engineer with Space Research Bölkow GmbH (1966-1969), Head of the Mechanics Division of MBB (1969-1976), Technical Assistant of L. Bölkow (1976-1978), and Managing Director of the Apparatus Development Section of MBB (1978-1982). Since 1982, he has been a full Professor of Mechanics at the Technical University of Munich. His teaching and research interests center around dynamics and control of rigid and elastic mechanical systems in general, and nonlinear dynamics, robotics, grasping, and walking in particular. He has published more than 100 articles and four books. 